\title{
Neuroblastoma de Alto Riesgo en Uruguay. ¿Dónde estamos?
}

DOI

\author{
High Risk Neuroblastoma in Uruguay. Where are we? \\ Neuroblastoma de Alto Risco no Uruguai. Onde estamos?
} Fabiana Morosini'; Gustavo Dufort'ㄹ Anaulina Silveira ${ }^{3}$ Mariela Castiglioni ${ }^{4}$; Carolina Pagés5; Elizabeth Simón ${ }^{5}$; Silvana Zuccolo ${ }^{5}$;
Andrea Incoronato ${ }^{5}$; Paloma Amarillo5; Inés Pereira ${ }^{5}$; Agustín Dabezies ${ }^{6}$; Luis Castillo

Resumen

Introduçáo: El neuroblastoma es el tumor sólido extracraneal más frecuente en niños. Aproximadamente el $50 \%$ de los pacientes son clasificados como de alto riesgo, con base en características clínicas, biológicas e histológicas. Objetivo: Describir a la población asistida en el Centro Hemato-Oncológíco Pediátrico (CHOP) del Centro Hospitalario Pereira Rossell (CHPR) con diagnóstico de neuroblastoma de alto riesgo, su tratamiento y sobrevida. Método: Estudio descriptivo y retrospectivo de todos los pacientes con neuroblastoma de alto riesgo diagnosticados en el CHOP entre el 2001 y el 2015. En el CHOP se ubica el Registro Nacional de Cáncer Pediátrico, así como también el Archivo de Historias Clínicas de todos los pacientes. Los datos son recolectados y analizados por el sector de estadística. Resultados: Se diagnosticaron 35 pacientes, de los cuales 20 (57\%) eran varones con mediana de edad de 36,6 meses (5-93), localización suprarrenal 23 (66\%) y 100\% estadio IV. Metástasis, médula ósea y hueso: 27 (71\%). Treinta y tres pacientes recibieron autotransplante de progenitores hematopoyéticos (TPH) (94\%). Estatus previo a TPH, remisión completa: 19 (58 \%), remisión parcial: 14 (42\%). Mortalidad relacionada al tratamiento: $15 \%$ y de recaídas: $68 \%$. Mediana de tiempo de recaída: 15 meses (3-52). La probabilidad de sobrevida global y sobrevida libre de eventos a 5 ańos fue de 37,8\% \pm 8,4 y 23,8\% 7,3 (mediana de seguimiento 40 meses). Conclusión: A pesar del tratamiento intensivo y de las medidas de soporte adecuadas, el pronóstico en los nińos con neuroblastoma de alto riesgo sigue siendo pobre en nuestro país. Es necesario incorporar nuevas estrategias terapéuticas aún no disponibles en nuestro medio.

Palabras clave: Neuroblastoma; Neoplasias; Niño; Terapéutica.

\begin{abstract}
Introduction: Neuroblastoma is the most common extracranial solid tumor in children. Approximately $50 \%$ of patients are classified as high risk on the basis of clinical, biological, and histological characteristics. Objective: To describe the population of patients diagnosed with high-risk neuroblastoma at the Centro Hemato-Oncológíco Pediátrico (CHOP, Center for Pediatric Hematology and Oncology) of the Centro Hospitalario Pereira Rossell (CHPR, Pereira Rossell Hospital), in terms of their treatment and survival. Method: Descriptive, retrospective study of all patients diagnosed with highrisk neuroblastoma at the CHOP between 2001 and 2015. The National Registry of Pediatric Cancer is located at the CHOP, as is the Archive of Patient Clinical Histories. The data are collected and analyzed by the statistics sector. Results: Among the 35 patients diagnosed, 20 (57\%) were men, the median age was 36.6 months (range, 5-93 months), and the tumor had an adrenal location in $23(66 \%)$. All of the tumors were classified as stage IV. Metastasis to the bone marrow or bone was seen in 27 (71\%). Thirty-three patients (94\%) received autologous hematopoietic stem-cell transplantation (HSCT). The status prior to HSCT was complete remission in 19 (58\%) and partial remission in $14(42 \%)$. The treatment-related mortality rate was $15 \%$, and the relapse rate was $68 \%$. The median time to relapse was 15 months (3-52 months). The probability of overall survival and 5-year event-free survival was $37.8 \% \pm 8.4$ and $23.8 \% \pm 7.3$ (median follow-up of 40 months), respectively. Conclusion: Despite intensive treatment and adequate support measures, the prognosis for high-risk neuroblastoma in children remains poor in Uruguay. There is a need to incorporate new therapeutic strategies not yet available in our country.

Key words: Neuroblastoma; Neoplasms; Child; Therapeutics.
\end{abstract}

\section{Resumo}

Introducción: $\mathrm{O}$ neuroblastoma é o tumor sólido extracraniano mais frequente em crianças. Aproximadamente $50 \%$ dos pacientes são classificados como de alto risco considerando as características clínicas, biológicas e histológicas. Objetivo: Descrever a população atendida no Centro Hemato-Oncológíco Pediátrico (CHOP) do Centro Hospitalario Pereira Rossell (CHPR) com diagnóstico de neuroblastoma de alto risco, seu tratamento e sobrevida. Método: Estudo descritivo, retrospectivo, de todos os pacientes com neuroblastoma de alto risco diagnosticados no CHOP, no período entre 2001 e 2015 . O Registro Nacional de Câncer Pediátrico está localizado no CHOP, bem como o Arquivo de Histórias Clínicas de todos os pacientes. Os dados são coletados e analisados pelo setor estatístico. Resultados: Foram diagnosticados 35 pacientes. Vinte (57\%) eram do sexo masculino. Mediana de idade: 36,6 meses (5-93). Localização suprarrenal: 23 (66\%). Estádio IV 100\%. Metástases, medula óssea e osso: 27 (71\%). Trinta e três pacientes receberam transplante de células-tronco hematopoiéticas (TCTH) (94\%). Status prévio a TCTH, remissão completa: 19 (58\%), remissão parcial: 14 (42\%). Incidência de mortalidade relacionada ao tratamento: $15 \%$ e de recaídas: $68 \%$. Mediana do tempo de recaída: 15 meses (3-52). A probabilidade de sobrevida global e sobrevida livre de eventos aos cinco anos foi de $37,8 \% \pm 8,4$ e $23,8 \% \pm 7,3$ (mediana de seguimento 40 meses). Conclusáo: Apesar do tratamento intensivo e medidas de suporte adequadas, o prognóstico em crianças com neuroblastoma de alto continua sendo pobre no Uruguai. É necessário incorporar novas estratégias terapêuticas que ainda não estão disponíveis em nosso meio.

Palavras-chave: Neuroblastoma; Neoplasias; Criança; Terapêutica.

\footnotetext{
1 Pediatra. Centro Hemato-Oncológico Pediátrico (CHOP)/Centro Hospitaleiro Pereira Rossell (CHPR). Montevideo, Uruguay. E-mail: morosinifa@gmail.com.

2 Jefe de Unidad de Trasplante de Médula Ósea. CHOP/CHPR. Montevideo, Uruguay. E-mail: gustavo.dufort@gmail.com.

${ }^{3}$ Analista en Economía. CHOP/CHPR. Montevideo, Uruguay. E-mail: anaulina.silveira@gmail.com.

${ }^{4}$ Médico. CHOP/CHPR. Montevideo, Uruguay.E-mail: secretariamedica@fundacionperezscremini.com.uy.

${ }^{5}$ Pediatra. CHOP/CHPR. Montevideo, Uruguay.E-mail: secretariamedica@fundacionperezscremini.com.uy.

${ }^{6}$ Coordinador. CHOP/CHPR. Montevideo, Uruguay. E-mail: secretariamedica@fundacionperezscremini.com.uy.

${ }^{7}$ Director. CHOP/CHPR Montevideo, Uruguay.E-mail: secretariamedica@fundacionperezscremini.com.uy.

Dirección para correspondencia: Fabiana Morosini. Bvar Artigas 1556. CP 11200. Montevideo, Uruguay.
} 


\section{INTRODUCCIÓN}

El neuroblastoma es un tumor embrionario del sistema nervioso autónomo que tiene su origen en las células de la cresta neural ${ }^{1}$. Constituye el segundo tumor sólido más frecuente en la infancia, después de los tumores del sistema nervioso central, y el más frecuente en los niños menores de cinco ańos ${ }^{1-3}$.

Su comportamiento muy heterogéneo es bien conocido, variando desde un tumor sumamente agresivo que provoca gran morbimortalidad hasta un tumor con alta tasa de regresión espontánea y curación ${ }^{4,5}$. Aproximadamente la mitad de los niños con neuroblastoma debutan con enfermedad metastásica.

Se identifican diferentes grupos de riesgo en los pacientes con neuroblastoma al momento del diagnóstico; la mayoría de las clasificaciones tiene en cuenta factores clínicos (como la edad y el estadio de la enfermedad), patológicos (grado de diferenciación e índice de mitosis) y biológicos (amplificación del gen $N$-myc y otras alteraciones genéticas $)^{6,7}$. Los pacientes de mayor edad portadores de enfermedad metastásica con amplificación del gen $N$-myc tienen peor pronóstico y mayor probabilidad de muerte.

Las clasificaciones actuales dividen la enfermedad en riesgo bajo, intermedio o alto, según la edad, estadio, patología y alteraciones genéticas. Se considera enfermedad de alto riesgo la de los pacientes mayores de un año con estadio IV o la de los menores de un año con enfermedad en estadio IV y $N-m y c$ amplificado; la de pacientes con enfermedad en estadio III y $N-m y c$ amplificado o histología desfavorable o la de pacientes con estadio II, $N$-myc amplificado e histología desfavorable. Los pacientes con estadios localizados, menores de 1 año, siempre se consideran de riesgo bajo ${ }^{7,8}$.

La estrategia terapéutica en estos tumores de alto riesgo se divide en tres fases: inducción de la remisión con quimioterapia seguida de cirugía del tumor primario, consolidación con quimioterapia a altas dosis y rescate con trasplante autólogo de progenitores hematopoyéticos (TPH) y radioterapia, y tratamiento de la enfermedad residual con ácido 13 -cis retinoico ${ }^{5,9,10}$. Hay una correlación entre sobrevida e intensidad de tratamiento, lo que ha sido la base para la consolidación con quimioterapia mieloablativa y rescate con TPH autólogo ${ }^{11,12}$. A su vez, la respuesta al tratamiento de inducción tiene implicancias pronósticas ${ }^{13}$.

A pesar de la intensificación del tratamiento, la sobrevida a largo plazo en los pacientes con enfermedad de alto riesgo se mantiene en 30 a $40 \%$ 5,7,12,14. Incluso, a pesar de la respuesta inicial a la quimioterapia, 10 a $20 \%$ de los pacientes padecen progresión o refractariedad ${ }^{15,16}$.

Recientemente, nuevos tratamientos inmunológicos, como los anticuerpos antigangliósido 2 (anti-GD2), han sido incorporados mostrando mejoría en la sobrevida $1,5,10$. Publicaciones internacionales reportan sobrevida global y libre de eventos significativamente superiores al tratamiento estándar ${ }^{17}$.

El objetivo del estudio es describir a la población asistida durante 15 ańos consecutivos en el servicio de Hemato-Oncología Pediátrica (CHOP) del Centro Hospitalario Pereira Rossell (CHPR) con diagnóstico de neuroblastoma de alto riesgo, su tratamiento y sobrevida.

\section{MÉTODO}

Se realizó un estudio descriptivo y retrospectivo que incluyó a todos los niños de 0-14 años con diagnóstico de neuroblastoma de alto riesgo que fueron diagnosticados en el CHOP entre el 1/1/2001 y el 31/12/15.

En el CHOP se ubica el Registro Nacional de Cáncer Pediátrico, así como también el Archivo de Historias Clínicas de todos los pacientes atendidos en el centro. Los datos de las historias clínicas de los pacientes fueron recolectados por la Data Manager de la institución en una planilla Excel diseñada para el estudio y analizados en el sector de estadística, previa firma de consentimiento informado por parte del paciente o su cuidador, según corresponda.

El diagnóstico de neuroblastoma se estableció por histología e inmunohistoquímica en piezas quirúrgicas del tumor primario y/o metástasis al momento del diagnóstico o por la confirmación de infiltración de médula ósea por placas de células extrahematopoyéticas típicas.

El tumor primario se evaluó por tomografía computada o resonancia magnética. La extensión de la enfermedad se determinó mediante la realización de aspirado de médula ósea, tomografía de tórax, centellograma óseo con tecnecio 99 y centellograma con metaiodobencilguanidina (MIBG), según recomendaciones internacionales ${ }^{5,7}$.

La estadificación se realizó de acuerdo con el sistema internacional de estadificación de neuroblastoma (INSS), en estadios I a IV ${ }^{18}$.

Se consideró neuroblastoma de alto riesgo el de todos los pacientes mayores de un ańo con estadio IV y el de los pacientes con estadios menores con histología desfavorable y/o $N$-myc amplificado ${ }^{1,7}$.

La anatomía patológica fue informada como favorable o desfavorable, según los criterios de Shimada ${ }^{19}$. Se consideró histología desfavorable: tumor indiferenciado, alto índice de mitosis y cariorrexis (MKI) pobre en estroma.

La determinación de la amplificación del gen $N$-myc se realizó por técnica de hibridación in situ cromatográfica (CISH) cuando este estudio estuvo disponible.

Todos los pacientes fueron tratados con un esquema que incluyó inducción con quimioterapia y cirugía 
del tumor primario, consolidación con quimioterapia mieloablativa y auto TPH, radioterapia del sitio del tumor y/o metástasis y mantenimiento con ácido 13-cis retinoico (6 ciclos de 14 días de duración seguidos de 14 días de descanso durante 6 meses). El protocolo de quimioterapia de inducción utilizado entre 2001 y 2010 (protocolo A) fue seis ciclos de: vincristina $\left(2 \mathrm{mg} / \mathrm{m}^{2}\right.$ día 1$)$, ifosfamida (3.000 $\mathrm{mg} / \mathrm{m}^{2} /$ día días 1 y 2$)$, adriamicina $\left(50 \mathrm{mg} / \mathrm{m}^{2}\right.$ día 3), cisplatino $\left(100 \mathrm{mg} / \mathrm{m}^{2}\right.$ día 3). Posteriormente, se siguió el protocolo $\mathrm{B}$, que consta de seis ciclos de quimioterapia (ciclo 1 y 2: ciclofosfamida $400 \mathrm{mg} / \mathrm{m}^{2} /$ día + topotecan 1,2 $\mathrm{mg} / \mathrm{m}^{2} /$ día días 1 a 5 ; ciclos 3 y 5 : cisplatino $50 \mathrm{mg} / \mathrm{m}^{2} /$ día días 1 a 4 + etopósido $200 \mathrm{mg} / \mathrm{m}^{2} /$ día días 1 a 3 ; ciclos 4 y 6: ciclofosfamida $2,1 \mathrm{~g} / \mathrm{m}^{2} /$ día días 1 y $2+$ doxorrubicina $25 \mathrm{mg} / \mathrm{m}^{2} /$ día días 1 a 3 + vincristina $0,67 \mathrm{mg} / \mathrm{m}^{2} /$ día días 1 a 3). En los pacientes con peso menor a 12 kilos la dosis de citostáticos se ajustó al peso. Se realizó soporte con factores de crecimiento post quimioterapia y reposición con hemoderivados de acuerdo con requerimientos, así como profilaxis de la infección por Pneumocystis jirovecii con trimetroprim-sulfametoxazol tres veces por semana.

El estatus previo al trasplante se definió según los criterios internacionales de respuesta ${ }^{7}$. Respuesta completa (RC): sin evidencia de tumor primario ni metástasis. Muy buena respuesta parcial (MBRP): reducción de más del 90 $\%$ del tumor primario, sin evidencia de metástasis (excepto ósea), sin nuevas lesiones óseas, con mejoría de lesiones preexistentes en centellograma óseo. Respuesta parcial (RP): reducción entre 50-90\% del tumor primario, 50 $\%$ o más de reducción en sitios metastásicos, disminución en número de metástasis óseas en $50 \%$.

Los progenitores hematopoyéticos para el trasplante autólogo fueron obtenidos de sangre periférica por leucaféresis, post movilización con factores de crecimiento granulocitario; la oportunidad para la estimulación y recolección de progenitores se planificó en cada paciente en forma individual, entre el segundo y cuarto ciclo de quimioterapia. Se determinó un mínimo de $2 \times 10^{6}$ células CD34+/kg para realizar la infusión.

Los regímenes de condicionamiento utilizados fueron CEM: carboplatino $\left(425 \mathrm{mg} / \mathrm{m}^{2} /\right.$ día días $\left.-7,-6,-5,-4\right)$, etopósido $\left(400 \mathrm{mg} / \mathrm{m}^{2} /\right.$ día días $\left.-7,-6,-5,-4\right)$ y melfalán $\left(70 \mathrm{mg} / \mathrm{m}^{2} /\right.$ día días $\left.-7,-6,-5\right)$ hasta el año 2009 , y posteriormente BUMEL: busulfán $(1 \mathrm{mg} / \mathrm{kg}$ v/o c/6h días -8 a -5$)$ y melfalán $\left(70 \mathrm{mg} / \mathrm{m}^{2} /\right.$ día días -4 y -3$)$.

Se realizó profilaxis del síndrome de obstrucción sinusoidal con heparina en infusión continua intravenosa, profilaxis de la reactivación de citomegalovirus y herpes virus con aciclovir intravenoso y profilaxis de infecciones fúngicas con fluconazol. Se indicó trimetroprim sulfametoxazol como profilaxis de la infección por $P$. jirovecii en todos los pacientes. Los factores estimulantes del crecimiento de colonias granulocitarias se indicaron a partir del día +5 . La reposición de hemoderivados se realizó con productos desleucotizados e irradiados. Se definió injerto como el número absoluto de neutrófilos mayor de $500 / \mathrm{mm}^{3}$ por 3 días consecutivos.

La radioterapia se realizó en el lecho tumoral post TPH y las metástasis activas en la evaluación previa al $\mathrm{TPH}$, con la dosis recomendada de 2.160 cGy dividida en 12 fracciones.

Posteriormente, se realizó tratamiento de la enfermedad residual con ácido 13 cis-retinoico, $160 \mathrm{mg} / \mathrm{m} 2 /$ día vía oral, durante 6 ciclos mensuales de 14 días cada uno.

El seguimiento se realizó hasta el 30/12/16 o la fecha de fallecimiento (lo que sucediera primero).

Se presentan tablas y gráficos de frecuencias para la descripción de variables cualitativas, así como medidas de resumen para las continuas. Los cálculos de sobrevida se realizaron por el método de Kaplan-Meier. En los casos de comparación de sobrevida se utilizó test Log Rank. En todos los casos se fijó un nivel de significación de 0,05.

El estudio fue avalado por la Dirección Pediátrica del CHPR y el Comité de Ética Hospitalario. Todos los pacientes firmaron un consentimiento informado al iniciar el tratamiento.

\section{RESULTADOS}

Se diagnosticaron 1.484 casos de cáncer en nińos menores de 14 años en el CHOP durante el periodo comprendido en el estudio; de éstos, 75 fueron neuroblastomas y 35 fueron clasificados como de alto riesgo.

En la Tabla 1 se muestran las características de la población: el $57 \%$ fueron varones, con una media de edad de 36,6 \pm 3,1 meses (rango 5 a 93 meses). El tumor primario se localizó en las glándulas suprarrenales en el 66 $\%$ de los casos. Todos los pacientes presentaron metástasis en el momento del diagnóstico, correspondiente a estadio IV de INSS; 27 de ellos en el hueso y la médula ósea.

En 14 pacientes se realizó biopsia del tumor primario, en cinco de ellos la histología se informó como desfavorable, en tres favorable y en seis no fue específica. La amplificación del gen $N$-myc se estudió en nueve pacientes, fue positiva en cuatro.

El tratamiento recibido se observa en la Tabla 2. Todos los pacientes recibieron quimioterapia. La cirugía se realizó en 31 pacientes ( 2 fallecieron previo al TPH, en un paciente no se evidenció tumor primario resecable, en otro no se identificó tumor residual en evaluación imagenológica post inducción).

Recibieron TPH 33 pacientes (2 no lo recibieron por enfermedad progresiva y muerte previa al TPH). Como se muestra en la Tabla 3, el $54 \%$ de estos pacientes 
Tabla 1. Características de pacientes con neuroblastoma de alto riesgo diagnosticados en el $\mathrm{CHOP}(\mathrm{n}=35)$

\begin{tabular}{l|c|c}
\multicolumn{1}{c|}{ Sexo masculino } & N & $\%$ \\
\hline Sexo masculino & 20 & 57 \\
\hline Sexo femenino & 15 & 43 \\
\hline Localización tumor primario & & \\
\hline Suprarrenal & 23 & 66 \\
\hline Paravertebral & 11 & 31 \\
\hline Desconocido & 1 & 3 \\
\hline Metástasis & 35 & 100 \\
\hline Sólo MO & 3 & 9 \\
\hline Sólo ósea & 2 & 9 \\
\hline MO + ósea & 27 & 71 \\
\hline Otras & 7 & 20 \\
\hline Estadio INSS IV & 35 & 100 \\
\hline Histología (n=14) & & \\
\hline Desfavorable & 5 & 36 \\
\hline Favorable & 3 & 21 \\
\hline No específica & 6 & 43 \\
\hline
\end{tabular}

Tabla 2. Tratamiento de pacientes con neuroblastoma de alto riesgo diagnosticados en el $\mathrm{CHOP}(n=35)$

\begin{tabular}{l|c|c}
\hline \multicolumn{1}{c|}{ Tratamiento recibido } & N & $\%$ \\
\hline Quimioterapia & & \\
\hline$\quad$ Protocolo A § & 14 & \\
\hline$\quad$ Protocolo B* & 21 & \\
\hline Cirugía & & \\
\hline$\quad$ Total & 24 & 69 \\
\hline$\quad$ Parcial & 7 & 20 \\
\hline Sin Cirugía & 4 & 11 \\
\hline TPH autólogo & 33 & 94 \\
\hline Radioterapia & & \\
\hline$\quad$ Lecho quirúrgico & 28 & 80 \\
\hline$\quad$ Metástasis & 6 & 17 \\
\hline
\end{tabular}

\$Protocolo A: vincristina + adriamicina + ifosfamida + cisplatino (dosis en texto). *Protocolo B: ciclo 1 y 2: ciclofosfamida + topotecan 1; ciclos 3 y 5 : cisplatino + etopósido; ciclos 4 y 6: ciclofosfamida + doxorrubicina + vincristina (dosis en texto).

se encontraban en remisión completa al momento del procedimiento. La mediana del número de células CD34+ infundidas fue de $8,37 \pm 0,84 \times 10^{6} / \mathrm{kg}$ y la mediana de días para el injerto fue de 11. A partir del año 2010, 14 pacientes recibieron condicionamiento con busulfán y melfalán.

Veintiocho pacientes recibieron radioterapia en el lecho tumoral y seis en sitios metastásicos después del $\mathrm{TPH}$. Cinco pacientes no la recibieron porque fallecieron durante el TPH.
Tabla 3. Consolidación con auto TPH en pacientes con neuroblastoma de alto riesgo diagnosticados en el CHOP $(n=33)$

\begin{tabular}{|c|c|c|}
\hline Tratamiento recibido & $\mathbf{N}$ & $\%$ \\
\hline \multicolumn{3}{|l|}{ Respuesta a inducción } \\
\hline RP/MBRP & $12 / 2$ & 43 \\
\hline RC & 19 & 57 \\
\hline \multicolumn{3}{|l|}{$\mathrm{N}^{\circ}$ de aféresis pre TPH } \\
\hline 1 & 31 & 94 \\
\hline 2 & 2 & 6 \\
\hline $\begin{array}{l}N^{\circ} \text { de CD34 infundidas } \\
\left(\times 10^{6} / \mathrm{kg}\right)\end{array}$ & \multicolumn{2}{|c|}{$\begin{array}{c}\text { Mediana: } 8,37 \pm 0,84 \\
\text { Mínimo } 3,11 ; \\
\text { máximo } 26,11\end{array}$} \\
\hline \multicolumn{3}{|l|}{ Condicionamiento } \\
\hline CEM & 19 & 58 \\
\hline BUMEL & 14 & 42 \\
\hline $\begin{array}{l}\text { Día RAN mayor de } 500 / \mathrm{mm}^{3} \text {, } \\
\text { mediana (rango) }\end{array}$ & \multicolumn{2}{|c|}{$\begin{array}{c}11 \\
(9-70)\end{array}$} \\
\hline MRT & 5 & 15 \\
\hline
\end{tabular}

TPH: trasplante de progenitores hematopoyéticos; RAN: recuento absoluto de neutrófilos; MRT: mortalidad relacionada al trasplante; CEM: carboplatino etopósido melfalán; BUMEL: busulfán melfalán

Todos los pacientes que sobrevivieron post TPH recibieron 6 ciclos de ácido retinoico para tratamiento de la enfermedad residual, sin complicaciones.

La incidencia acumulada de recaídas fue de 67,9 \% (19 pacientes de 28 que sobrevivieron post TPH); la mediana de tiempo de recaída fue de 15 meses desde el TPH, con un rango de tres a 52 meses.

Veintiún pacientes fallecieron: en 16 casos fue por enfermedad progresiva ( 2 de ellos previo al TPH) y en cinco, por causas relacionadas al tratamiento. La incidencia de mortalidad relacionada al tratamiento fue de $15 \%$, como se ve en la Figura 1. No se registraron muertes por toxicidad durante la inducción; todas fueron por toxicidad relacionada al TPH (4 sepsis con fallo multiorgánico, 1 síndrome de obstrucción sinusoidal) entre tres días y tres meses post TPH. En todos los casos, en pacientes que habían recibido condicionamiento con CEM.

Un paciente desarrolló un síndrome mielodisplásico con monosomía siete un año post TPH. Recibió un TPH de sangre de cordón umbilical no relacionada y se encuentra vivo, sin enfermedad, en el momento actual.

Con una mediana de seguimiento de 40 meses (rango 5-157 meses), la sobrevida libre de eventos (SLE) fue del $23,8 \% \pm 7,3$ a los cinco ańos y la sobrevida global (SG), del $37,8 \% \pm 8,4$ (Figura 1).

La SG en el grupo de pacientes con RC previo al TPH fue del 44,4\% $\%$ 11,7 en comparación con el 35,2\% $\%$ 13,2 en el grupo de pacientes con MBRP y RP $(\mathrm{p}=0,352)$ (Figura 2). 

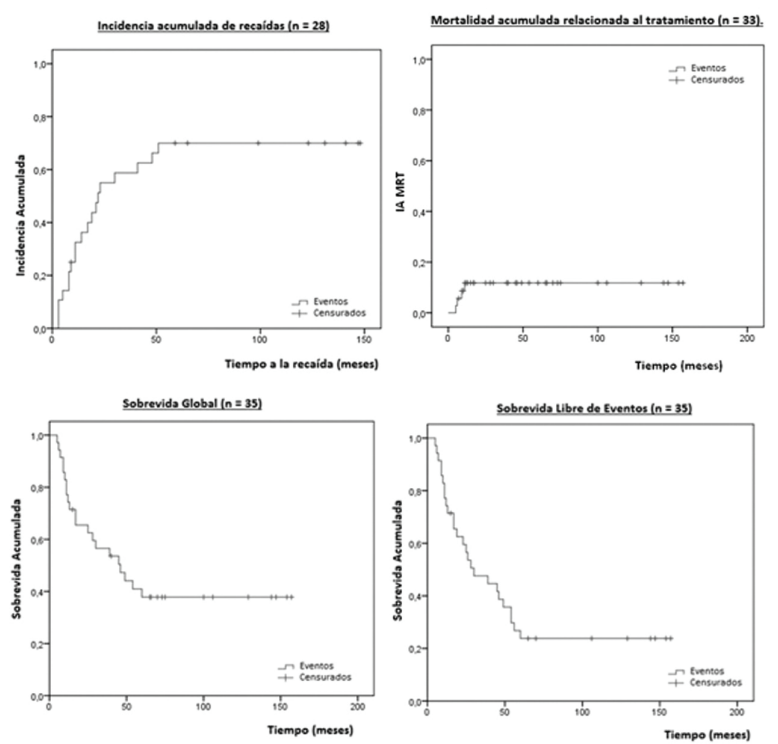

Figura 1. Recaída, mortalidad relacionada al trasplante, sobrevida global y sobrevida libre de eventos en pacientes con neuroblastoma de alto riesgo

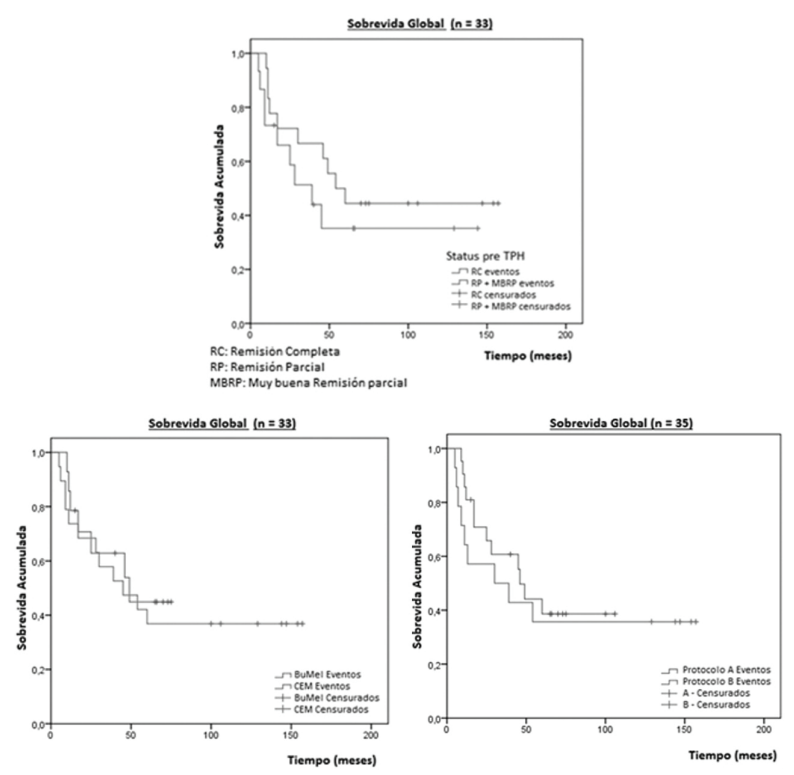

RC: Respuesta completa; RP: respuesta parcial; MBRP: muy buena respuesta parcial

Figura 2. Sobrevida global en pacientes con neuroblastoma de alto riesgo según respuesta a inducción, quimioterapia de inducción y condicionamiento (RC vs. RP + MBRP)

La sobrevida en los niños que recibieron inducción con protocolo A fue del 38,6\% $\% \pm 0,11$ frente al 35,7 $\% \pm 12,8$ en los que recibieron protocolo $B(p=0,540)$. A su vez, la sobrevida en los pacientes que recibieron condicionamiento con BUMEL fue del 44,9\% $\%$ 14,3 en comparación con el 36,8\% $\%$ 11,1 en los que recibieron CEM $(\mathrm{p}=0,616)$ (Figura 2).
De los 19 pacientes que recayeron, cinco sobreviven al finalizar el estudio: tres continúan vivos, en remisión a los 18 meses, tres y cinco años post recaída respectivamente, y dos pacientes continúan con tratamiento multimodal paliativo con enfermedad estable. De los tres pacientes que se encuentran en remisión, dos recibieron quimioterapia y segundo auto TPH con RC posterior y el tercero recibió quimioterapia e inmunoterapia con anti-GD2.

\section{DISCUSIÓN}

El neuroblastoma correspondió al $5 \%$ de los casos de cáncer diagnosticados en los niños asistidos en el $\mathrm{CHOP}$ en el periodo del estudio, lo que concuerda con lo reportado en otros países ${ }^{3}$. Es una enfermedad de la infancia, con un promedio de edad en este estudio de 36 meses, como en otras publicaciones ${ }^{20}$.

La totalidad de los pacientes con diagnóstico de neuroblastoma de alto riesgo correspondieron a niños con enfermedad en estadio IV. Esto correspondió al $50 \%$ de los nińos diagnosticados con neuroblastoma en el periodo, lo que concuerda con lo reportado internacionalmente. La localización fue abdominal en todos los pacientes, excepto en uno en el que el sitio del tumor primario fue desconocido. En otros estudios que incluyen mayor cantidad de pacientes, se identifican pacientes con estadios menores y enfermedad de alto riesgo que presentan una mejor sobrevida con respecto a los que debutan con enfermedad avanzada ${ }^{11}$.

La amplificación del gen $N$-myc es un factor de mal pronóstico en los pacientes portadores de neuroblastoma ${ }^{5,7}$. En este estudio, sólo pudo realizarse en 9 pacientes por no ser accesible la técnica en nuestro medio durante algunos periodos. Esto es una limitación, no pudiéndose analizar el impacto de este factor de riesgo en nuestra casuística. Actualmente se busca en todos los pacientes con neuroblastoma, otros marcadores pronósticos como la pérdida de heterocigocidad del cromosoma $11 \mathrm{o}$ la presencia de mutaciones en ALK no se encuentran disponibles actualmente en nuestro país.

La estrategia de tratamiento en el CHOP es posible debido a la disponibilidad de recursos necesarios para la realización de TPH, el desarrollo de cuidados de soporte que permiten la indicación de quimioterapia intensa y la capacitación técnica de los recursos humanos en dichos procesos.

La quimioterapia de inducción se realizó con dos protocolos ya descritos de acuerdo con el utilizado en el momento del diagnóstico. Dichos protocolos se basan en la utilización de platinos y antraciclínicos; más recientemente se incorporaron topotecan y ciclofosfamida a estos protocolos ${ }^{21}$. 
El TPH es parte de la estrategia de tratamiento de estos pacientes; las comunicaciones internacionales se basan en protocolos que incluyen este tratamiento. Varios ensayos controlados randomizados han demostrado mejoría en la SLE en los niños que recibieron TPH (31\% a $47 \%$ ) en comparación con quimioterapia convencional (22\% a $31 \%)^{20}$. El beneficio del TPH se mantiene en la SLE, no teniendo tanta incidencia en la SG ${ }^{15}$. El TPH se realizó en 33 pacientes, la mortalidad relacionada al trasplante fue mayor que la reportada en otras series ${ }^{11,12,21}$.

El número de células CD34+ infundidas fue acorde al objetivo propuesto y no fue necesario realizar más de dos aféresis en ningún paciente. La mediana de días para el injerto fue de 11, concordante con lo comunicado en otras series de pacientes, lo que permite una menor exposición a riesgo de infección y otras complicaciones de internaciones prolongadas en estos pacientes.

Publicaciones internacionales han reportado mejor sobrevida y menor toxicidad con condicionamiento BUMEL en comparación con $\mathrm{CEM}^{22-24}$. Si bien en nuestro estudio la sobrevida fue mayor en los pacientes que recibieron condicionamiento con BUMEL, esta diferencia no fue significativa. Es necesario continuar analizando a los pacientes con neuroblastoma tratados en nuestro servicio para confirmar este hallazgo. Actualmente los niños con neuroblastoma reciben tratamiento mieloablativo con BUMEL previo al TPH en todos los casos.

Un porcentaje de pacientes recaen luego del tratamiento de inducción y consolidación aun habiendo alcanzado respuesta clínica completa, lo que indica la presencia de enfermedad residual resistente al tratamiento. El tratamiento con ácido retinoico de la enfermedad residual ha demostrado mejoría en la SLE en algunos estudios ${ }^{15} \mathrm{y}$ forma parte del tratamiento recomendado post consolidación dirigido a erradicar la enfermedad residual.

A pesar de la intensificación del tratamiento y las mejoras en el soporte, los niños con neuroblastoma de alto riesgo continúan teniendo una sobrevida baja. En este grupo de pacientes, la SG fue similar a lo reportado internacionalmente en pacientes que recibieron protocolos de tratamiento similares ${ }^{12}$, aunque el tiempo de seguimiento es menor.

Los últimos ensayos clínicos en neuroblastoma de alto riesgo incorporan la utilización de anticuerpos antiGD2 (anticuerpo quimérico murino-humano ch14.18 o dinutuximab), presentes normalmente en algunos tejidos y también en las células de neuroblastoma, con o sin factores de crecimiento granulocitario e interleucina $2^{25}$, con lo cual se ha reportado una mejoría en la sobrevida con respecto al tratamiento convencional ${ }^{17}$. Por lo tanto, el tratamiento post consolidación de la enfermedad residual con anti-GD2, con o sin factores de crecimiento e interleucina 2 , se considera el estándar de tratamiento del neuroblastoma de alto riesgo en el momento actual. Estas opciones terapéuticas aún no están disponibles en nuestro país ni en la región, principalmente debido a su alto costo, a pesar de haber demostrado impacto positivo en la sobrevida de estos pacientes.

Si bien al comparar la sobrevida entre pacientes trasplantados en RC frente a RP la diferencia no fue significativa, el porcentaje de sobrevida es mayor en los pacientes en $\mathrm{RC}$, lo cual apoya la respuesta a la inducción como un factor pronóstico.

La principal causa de mortalidad en este grupo fue la enfermedad progresiva, lo que sigue confirmando el carácter agresivo de esta enfermedad. Ningún paciente falleció por toxicidad durante la inducción; la mortalidad relacionada al tratamiento se evidenció en los primeros tres meses post TPH, en todos los casos en pacientes que habían recibido condicionamiento con CEM.

Es necesario continuar este seguimiento para definir exactamente la sobrevida a largo plazo de estos pacientes, a medida que además se puedan incorporar nuevos tratamientos.

\section{CONCLUSIÓN}

En Uruguay los resultados del tratamiento del neuroblastoma de alto riesgo con la estrategia analizada son similares a los publicados por otros grupos. Sin embargo, a pesar de la intensificación en el tratamiento y la mejora en cuidados de soporte, el pronóstico sigue siendo pobre. Es necesario incorporar nuevos tratamientos aún no disponibles, que han demostrado un impacto favorable sobre los resultados.

\section{CONTRIBUCIONES DE LOS AUTORES}

Gustavo Dufort y Fabiana Morosini contribuyeron en la concepción y planeación del proyecto de investigación, en la obtención y/o análisis e interpretación de los datos y en la redacción y revisión crítica. Luis Castillo, en la redacción y revisión crítica. Anaulina Silveira, en el análisis e interpretación de datos. El resto de los autores, en la obtención y análisis de los datos.

\section{DECLARACIÓN DE CONFLICTO DE INTERESES}

Nada que Declarar.

\section{REFERENCIAS}

1. $\mathrm{PDQ}^{\circ}$ Pediatric Treatment Editorial Board. PDQ Neuroblastoma Treatment [Internet]. Bethesda, MD: National Cancer Institute; [acesso em 2017 febrero 1]. Disponible en: http://www.cancer.gov/types/ neuroblastoma/hp/neuroblastoma-treatment-pdq. 
2. Klaassen R, Trebo M, Koplewitz R, Weitzman S, Calderwood S. High-risk neuroblastoma in Ontario: a report of experience from 1989 to 1995 . J Pediatr Hematol Oncol. 2003;25(1):8-13.

3. Ries LAG, Smith MA, Gurney JG, Linet M, Tamra T, Young JL, Bunin GR. Cancer Incidence and Survival among Children and Adolescents: United States SEER Program 1975-1995, National Cancer Institute, SEER Program. NIH Pub. 1999;99:4649.

4. Yamamoto K, Hanada R, Kikuchi A, Ichikawa M, Aihara T, Oguma E, Moritani T, Shimanuki Y, Tanimura M, Hayashi Y. Spontaneous regression of localized neuroblastoma detected by mass screening. J Clin Oncol. 1998;16(4):1265-9.

5. Maris J. Recent advances in neuroblastoma. N Engl J Med. 2010;362(23): 2202-11.

6. Horner MJ, Ries LA, Krapcho M, et al. SEER Cancer Statistics Review, 1975-2006 [Internet]. Bethesda, MD: National Cancer Institute; [acesso em 2017 febrero 10]. Disponible en: https://seer.cancer.gov/archive/ csr/1975_2006.

7. Brodeur GM, Pritchard J, Berthold F, Carlsen NL, Castel V, Castelberry RP, De Bernardi B, et al. Revisions of the international criteria for neuroblastoma diagnosis, staging, and response to treatment. J Clin Oncol. 1993;11(8):1466-77.

8. Cohn SL, Pearson AD, London WB, Monclair T, Ambros PF, Brodeur GM, et al. The International Neuroblastoma Risk Group (INRG) classification system: an INRG Task Force report. J Clin Oncol. 2009;27(2):289-97.

9. Cheung NV, Geller G. Chemotherapy dose intensity correlates strong with response, median survival and median progression free survival in metastatic neuroblastoma. J Clin Oncol. 1991;9:1050-58.

10. Navin R, Pinto NR, Applebaum MA, Volchenboum SL, Matthay KK, London WB, Ambros PF, Nakagawara A, Berthold F, Schleiermacher G, Park JR, Valteau-Couanet D, Pearson AD, Cohn SL. Advances in Risk Classification and Treatment Strategies for Neuroblastoma. J Clin Oncol. 2015;33(27):3008-17.

11. Proust-Houdemont S, Pasqualini C, Blanchard P, Dufour C, Benhamou E, Goma G, Semeraro M, Raquin MA, Hartmann O, Valteau-Couanet D. Busulfan-melphalan in high-risk neuroblastoma: the 30-year experience of a single institution. Bone Marrow Transplant. 2016;51(8):1076-81.

12. Matthay KK, Reynolds CP, Seeger RC, Shimada H, Adkins ES, Haas-Kogan D, et al. Long-term results for children with high-risk neuroblastoma treated on a randomized trial of myeloablative therapy followed by 13-cis-retinoic acid: a children's oncology group study. J Clin Oncol. 2009;27:1007-1013.
13. Matthay K, Edeline V, Lumbroso J, Tanguy ML, Asselain B, Zucker JM, Valteau-Couanet D, Hartmann $\mathrm{O}$, Michon J. Correlation of early metastatic response by $123 \mathrm{I}$-metaiodobenzylguanidine scintigraphy with overall response and event-free survival in stage IV neuroblastoma. J Clin Oncol. 2003;21(13):2486-91.

14. Garaventa A, Rondelli R, Lanino E, Dallorso S, Dini G, Bonetti F, Arrighini A, Santoro N, Rossetti F, Miniero R, Andolina M, Amici A, Indolfi P, Lo Curto M, Favre C, Paolucci P, Pession A, De Bernardi B. Myeloablative therapy and bone marrow rescue in advanced neuroblastoma. Report from the Italian Bone Marrow Transplant Registry. Italian Association of Pediatric Hematology-Oncology, BMT Group. Bone Marrow Transplant. 1996;18(1):125-30.

15. Matthay KK, Villablanca JG, Seeger RC. Treatment of high-risk neuroblastoma with intensive chemotherapy, radiotherapy, autologous bone marrow transplantation, and 13-cis-retinoic acid. Children's Cancer Group. N Engl J Med. 1999;341(16):1165-73.

16. Frappaz D, Michon J, Coze C, Berger C, Plouvier E, Lasset C, Bernard JL, Stephan JL, Bouffet E, Buclon M, Combaret V, Fourquet A, Philip T, Zucker JM. LMCE3 treatment strategy: results in 99 consecutively diagnosed stage 4 neuroblastomas in children older than 1 year at diagnosis. J Clin Oncol. 2000;18(3):468-76.

17. Yu AL, Gilman AL, Ozkaynak MF, London WB, Kreissman SG, Chen HX, et al. Anti-GD2 antibody with GM-CSF, interleukin-2, and isotretinoin for neuroblastoma. N Engl J Med. 2010;363(14):1324-1334.

18. Brodeur GM, Seeger RC, Barrett A, et al. International criteria for diagnosis, staging, and response to treatment in patients with neuroblastoma. J Clin Oncol. 1988;6(12):1874-81.

19. Shimada H, Ambros I, Dehner L, Hata J, Joshi V, Roald B, et al. The International Neuroblastoma Pathology Classification (the Shimada System). Cancer. 1999;86(2):364-72.

20. London WB, Castleberry RP, Matthay KK, Look AT, Seeger RC, Shimada H, et al. Evidence for an age cutoff greater than 365 days for neuroblastoma risk group stratification in the Children's Oncology Group. J Clin Oncol. 2005;23(27):6459-65.

21. Park JR, Stewart CF, London WB, Santana VM, Shaw PJ, Cohn SL, et al. A topotecan-containing induction regimen for treatment of high risk neuroblastoma. J Clin Oncol. 2006;24:67.

22. Valteau-Couanet D, Le Deley MC, Bergeron C, Ducassou S, Michon J, Rubie H, et al. Long-term results of the combination of the N7 induction chemotherapy and the busulfan-melphalan high dose chemotherapy. Pediatr Blood Cancer. 2014;61(6):977-81.

23. Berthold F, Boos J, Burdach S, Erttmann R, Henze G, Hermann J, et al. Myeloablative megatherapy with 
autologous stem-cell rescue versus oral maintenance chemotherapy as consolidation treatment in patients with high-risk neuroblastoma: a randomised controlled trial. Lancet Oncol. 2005;6(9):649-58.

24. Pritchard J, Cotterill SJ, Germond SM, Imeson J, de Kraker J, Jones DR. High dose melphalan in the treatment of advanced neuroblastoma: results of a randomised trial (ENSG-1) by the European Neuroblastoma Study Group. Pediatr Blood Cancer. 2005;44(4):348-57.

25. Ozkaynak MF, Sondel PM, Krailo MD, Gan J, Javorsky B, Reisfeld RA, et al. Phase I study of chimeric human/murine anti-ganglioside GD2 monoclonal antibody (ch14.18) with granulocytemacrophage colony-stimulating factor in children with neuroblastoma immediately after hematopoietic stemcell transplantation: a children's cancer group study. J Clin Oncol. 2000;18(24):4077-4085.

Recebido em 28/12/2017

Aprovado em 5/4/2018 